\section{뉸 $\mathbb{N}_{\text {I }}$ IUCrData}

ISSN 2414-3146

Received 9 February 2018

Accepted 14 February 2018

Edited by W. T. A. Harrison, University of Aberdeen, Scotland

Keywords: crystal structure; pyrido[2,3-b]pyrazine.

\section{CCDC reference: 1823956}

Structural data: full structural data are available from iucrdata.iucr.org

\section{7-Bromo-1,4-bis(prop-2-ynyl)pyrido[2,3-b]- pyrazine-2,3(1H,4H)-dione}

\author{
Meriem Sikine, ${ }^{\mathrm{a} *}$ Youssef Kandri Rodi, ${ }^{\mathrm{a}}$ Younes Ouzidan, ${ }^{\mathrm{a}}$ Jerry P. Jasinski, ${ }^{\mathrm{b}}$ \\ Manpreet Kaur ${ }^{b}$ and El Mokhtar Essassic
}

aLaboratoire de Chimie Organique Appliquée, Faculté des Sciences et Techniques, Université Sidi Mohammed Ben Abdellah, Fès, Morocco, ${ }^{\mathbf{b}}$ Department of Chemistry, Keene State College, 229 Main Street, Keene, NH 03435-2001, USA, and ' ${ }^{\mathrm{C}}$ aboratoire de Chimie Organique Hétérocyclique, Pôle de Compétences Pharmacochimie, Mohammed V University in Rabat, BP 1014, Avenue Ibn Batouta, Rabat, Morocco. *Correspondence e-mail: sikine.meriem@gmail.com

In the title compound, $\mathrm{C}_{13} \mathrm{H}_{8} \mathrm{BrN}_{3} \mathrm{O}_{2}$, the pyrido-pyrazine fused-ring system is essentially planar (r.m.s. deviation $=0.061 \AA$ A). The prop-2-ynyl moieties are twisted away from the ring system in opposite directions. In the crystal, a single weak $\mathrm{C}-\mathrm{H} \cdots \mathrm{O}$ interaction generates [010] chains and aromatic $\pi-\pi$ stacking interactions between the pyridine rings are observed.
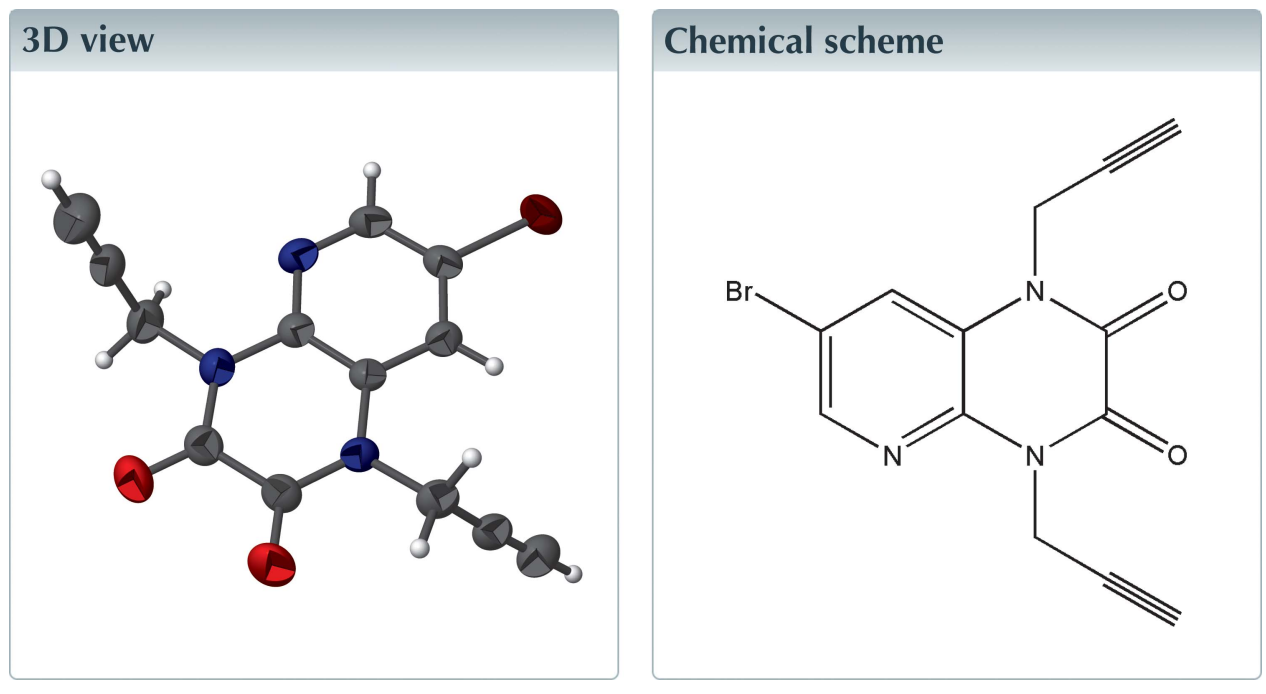

\section{Structure description}

Heterocycles containing a pyrido-pyrazine grouping possess useful medicinal properties (Zhang et al., 2012). They may also exhibit good inhibitory action on the corrosion of metals (Ouzidan et al., 2016). As part of our studies in this area, we now report the synthesis of a new pyrido[2,3- $b]$ pyrazine and its crystal structure.

The title compound crystallizes with one molecule in the asymmetric unit (Fig. 1). The pyrido-pyrazine moiety is essentially planar: the dihedral angle between the fused rings is $4.7(6)^{\circ}$. The prop-2-ynyl moieties are twisted away from the mean plane of the pyridopyrazine ring $\left[\mathrm{C} 8-\mathrm{C} 9-\mathrm{N} 2=114.0(2)^{\circ}\right.$ and $\left.\mathrm{C} 12-\mathrm{C} 11-\mathrm{N} 1=110.1(3)^{\circ}\right]$ to avoid steric repulsion. In the crystal, a single weak $\mathrm{C} 6-\mathrm{H} 6 \cdots \mathrm{O} 2$ interaction links the molecules into [010] chains (Fig. 2 and Table 1 ). In addition, weak $\pi-\pi$ stacking between the pyridine rings is observed [centroid-centroid separation $=3.7089$ (2) $\AA$ ] .

\section{Synthesis and crystallization}

To a solution of 7-bromopyrido[2,3-b]pyrazine-2,3(1H,4H)-dione $(0.2 \mathrm{~g}, 0.826 \mathrm{mmol})$, $\mathrm{K}_{2} \mathrm{CO}_{3}(0.456 \mathrm{~g}, 3.304 \mathrm{mmol})$, tetra- $n$-bromide butyl ammonium $(0.1 \mathrm{mmol})$ in DMF $(15 \mathrm{ml})$ was added propargyl bromide $(0.213 \mathrm{ml}, 1.790 \mathrm{mmol})$, and the mixture was stirred for $24 \mathrm{~h}$ at room temperature. After the solvent was evaporated under reduced pressure, 


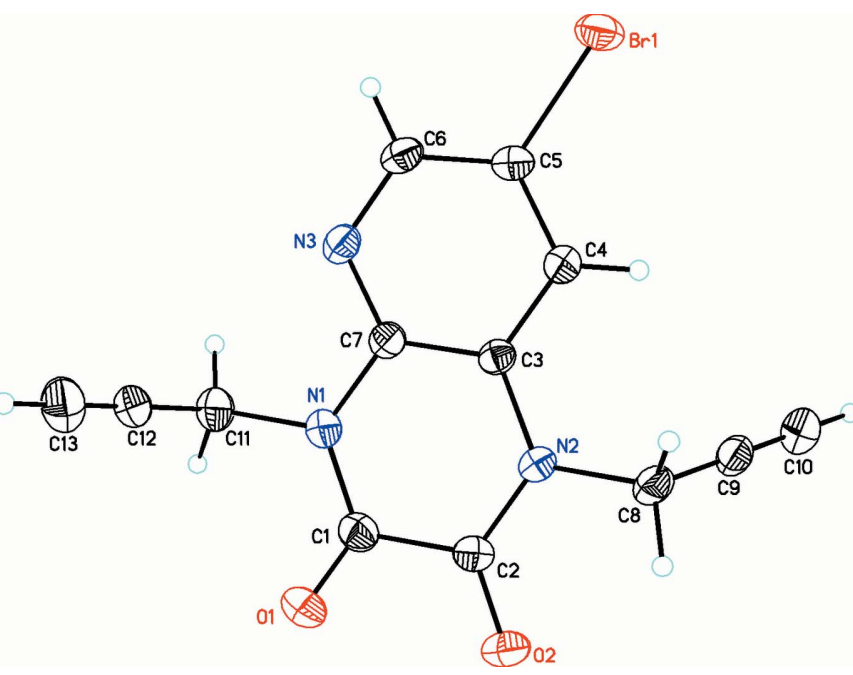

Figure 1

A view of the molecular structure, showing displacement ellipsoids drawn at the $30 \%$ probability level.

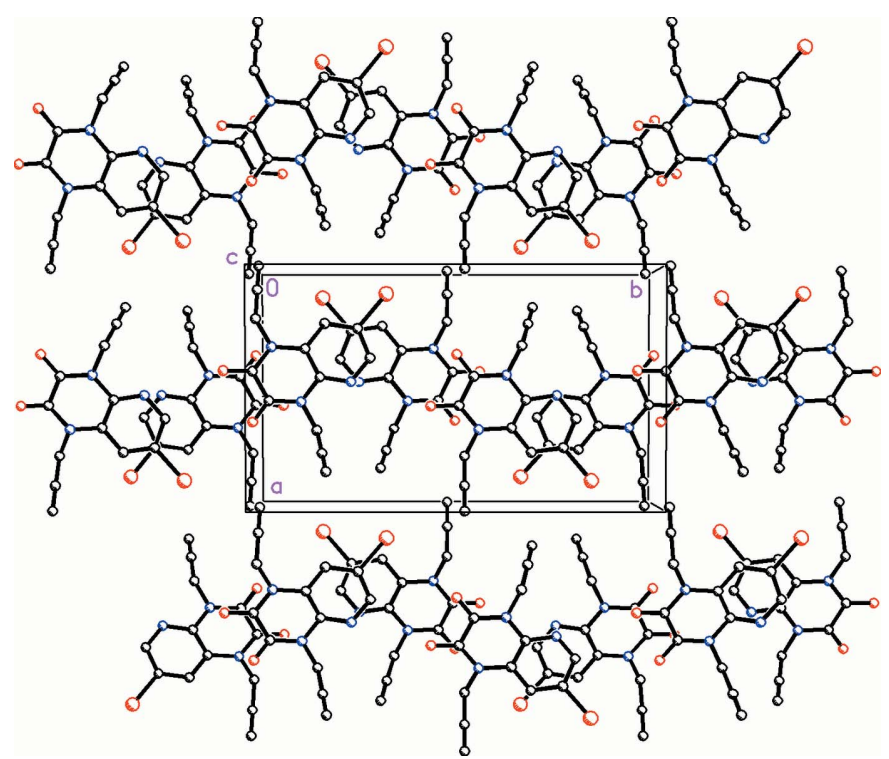

Figure 2

A partial view along the $c$ axis of the crystal packing. All $\mathrm{H}$ atoms except H6 have been omitted for clarity.

the product was isolated by chromatography on a silica gel column with ethyl acetate/hexane (1/3) as the eluent. Red crystals were isolated when the solvent was allowed to evaporate (yield $=18 \%$, m.p. $449 \mathrm{~K}$ ).

\section{Refinement}

Crystal data, data collection and structure refinement details are summarized in Table 2.
Table 1

Hydrogen-bond geometry $\left(\AA{ }^{\circ}\right)$.

\begin{tabular}{lllll}
\hline$D-\mathrm{H} \cdots A$ & $D-\mathrm{H}$ & $\mathrm{H} \cdots A$ & $D \cdots A$ & $D-\mathrm{H} \cdots A$ \\
\hline $\mathrm{C} 6-\mathrm{H} 6 \cdots \mathrm{O} 2^{\mathrm{i}}$ & 0.93 & 2.58 & $3.326(4)$ & 137 \\
\hline
\end{tabular}

Symmetry code: (i) $-x+1, y-\frac{1}{2},-z+\frac{1}{2}$.

Table 2

Experimental details.

\begin{tabular}{|c|c|}
\hline \multicolumn{2}{|l|}{ Crystal data } \\
\hline Chemical formula & $\mathrm{C}_{13} \mathrm{H}_{8} \mathrm{BrN}_{3} \mathrm{O}_{2}$ \\
\hline$M_{\mathrm{r}}$ & 318.13 \\
\hline Crystal system, space group & Monoclinic, $P 2_{1} / c$ \\
\hline Temperature $(\mathrm{K})$ & 293 \\
\hline$a, b, c(\AA)$ & $10.1922(5), 17.347(1), 7.0216(4)$ \\
\hline$\beta\left({ }^{\circ}\right)$ & $92.382(5)$ \\
\hline$V\left(\AA^{3}\right)$ & $1240.38(12)$ \\
\hline$Z$ & 4 \\
\hline Radiation type & $\mathrm{Cu} K \alpha$ \\
\hline$\mu\left(\mathrm{mm}^{-1}\right)$ & 4.55 \\
\hline Crystal size $(\mathrm{mm})$ & $0.22 \times 0.16 \times 0.1$ \\
\hline \multicolumn{2}{|l|}{ Data collection } \\
\hline Diffractometer & Rigaku Oxford Diffraction \\
\hline Absorption correction & $\begin{array}{l}\text { Multi-scan (CrysAlis PRO; Rigaku } \\
\text { OD, 2015) }\end{array}$ \\
\hline$T_{\min }, T_{\max }$ & $0.428,1.000$ \\
\hline $\begin{array}{l}\text { No. of measured, independent and } \\
\text { observed }[I>2 \sigma(I)] \text { reflections }\end{array}$ & $4477,2361,1994$ \\
\hline$R_{\text {int }}$ & 0.025 \\
\hline$(\sin \theta / \lambda)_{\max }\left(\AA^{-1}\right)$ & 0.614 \\
\hline \multicolumn{2}{|l|}{ Refinement } \\
\hline$R\left[F^{2}>2 \sigma\left(F^{2}\right)\right], w R\left(F^{2}\right), S$ & $0.036,0.101,1.06$ \\
\hline No. of reflections & 2361 \\
\hline No. of parameters & 172 \\
\hline $\mathrm{H}$-atom treatment & $\mathrm{H}$-atom parameters constrained \\
\hline$\Delta \rho_{\max }, \Delta \rho_{\min }\left(\mathrm{e} \AA^{-3}\right)$ & $0.50,-0.43$ \\
\hline
\end{tabular}

$\Delta \rho_{\max }, \Delta \rho_{\min }\left(\mathrm{e} \AA^{-3}\right) \quad 0.50,-0.43$

Computer programs: CrysAlis PRO (Rigaku OD, 2015), SHELXT (Sheldrick, 2015a), SHELXL2014 (Sheldrick, 2015b) and OLEX2 (Dolomanov et al., 2009).

\section{Funding information}

JPJ acknowledges the NSF-MRI program (grant No. CHE1039027) for funds to purchase the X-ray diffractometer.

\section{References}

Dolomanov, O. V., Bourhis, L. J., Gildea, R. J., Howard, J. A. K. \& Puschmann, H. (2009). J. Appl. Cryst. 42, 339-341.

Ouzidan, Y., Ouazzani Chahdi, F., Essassi, E. M. \& Hammouti, B. (2016). Pharma Chemica, 8, 85-95.

Rigaku OD (2015). CrysAlis PRO. Rigaku Oxford Diffraction, Yarnton, England.

Sheldrick, G. M. (2015a). Acta Cryst. A71, 3-8.

Sheldrick, G. M. (2015b). Acta Cryst. C71, 3-8.

Zhang, G., Liu, Y., Wang, S., Zhou, C., Huang, Q. \& Gong, P. (2012). Arch. Pharm. Pharm. Med. Chem. 345, 49-56. 


\section{full crystallographic data}

IUCrData (2018). 3, x180266 [https://doi.org/10.1107/S2414314618002663]

\section{7-Bromo-1,4-bis(prop-2-ynyl)pyrido[2,3-b]pyrazine-2,3(1H,4H)-dione}

Meriem Sikine, Youssef Kandri Rodi, Younes Ouzidan, Jerry P. Jasinski, Manpreet Kaur and El Mokhtar Essassi

7-Bromo-1,4-bis(prop-2-ynyl)pyrido[2,3-b]pyrazine-2,3(1H,4H)-dione

Crystal data

$\mathrm{C}_{13} \mathrm{H}_{8} \mathrm{BrN}_{3} \mathrm{O}_{2}$

$M_{r}=318.13$

Monoclinic, $P 2_{1} / c$

(000) $=632$

$a=10.1922(5) \AA$

$D_{\mathrm{x}}=1.704 \mathrm{Mg} \mathrm{m}^{-3}$

$a=10.1922(5) \AA$
$b=17.347(1) \AA$

$c=7.0216(4) \AA$

$\beta=92.382(5)^{\circ}$

$V=1240.38(12) \AA^{3}$

$\mathrm{Cu} K \alpha$ radiation, $\lambda=1.54184 \AA$

$Z=4$

Cell parameters from 1740 reflections

$\theta=4.3-71.4^{\circ}$

$\mu=4.55 \mathrm{~mm}^{-1}$

$T=293 \mathrm{~K}$

Prism, red

Data collection

Rigaku Oxford Diffraction model name?

$0.22 \times 0.16 \times 0.1 \mathrm{~mm}$

diffractometer

Radiation source: fine-focus sealed X-ray tube, Enhance (Cu) X-ray Source

Graphite monochromator

Detector resolution: 16.0416 pixels $\mathrm{mm}^{-1}$

$\omega$ scans

Absorption correction: multi-scan

(CrysAlis PRO; Rigaku OD, 2015)

Refinement

Refinement on $F^{2}$

Least-squares matrix: full

$R\left[F^{2}>2 \sigma\left(F^{2}\right)\right]=0.036$

$w R\left(F^{2}\right)=0.101$

$S=1.06$

2361 reflections

172 parameters

0 restraints

Primary atom site location: dual

Special details

Geometry. All esds (except the esd in the dihedral angle between two 1.s. planes) are estimated using the full covariance matrix. The cell esds are taken into account individually in the estimation of esds in distances, angles and torsion angles; correlations between esds in cell parameters are only used when they are defined by crystal symmetry. An approximate (isotropic) treatment of cell esds is used for estimating esds involving l.s. planes.
$T_{\min }=0.428, T_{\max }=1.000$

4477 measured reflections

2361 independent reflections

1994 reflections with $I>2 \sigma(I)$

$R_{\text {int }}=0.025$

$\theta_{\max }=71.3^{\circ}, \theta_{\min }=4.3^{\circ}$

$h=-12 \rightarrow 9$

$k=-20 \rightarrow 18$

$l=-8 \rightarrow 7$

Hydrogen site location: inferred from neighbouring sites

$\mathrm{H}$-atom parameters constrained

$w=1 /\left[\sigma^{2}\left(F_{\mathrm{o}}^{2}\right)+(0.0557 P)^{2}+0.1537 P\right]$

where $P=\left(F_{\mathrm{o}}^{2}+2 F_{\mathrm{c}}{ }^{2}\right) / 3$

$(\Delta / \sigma)_{\max }<0.001$

$\Delta \rho_{\max }=0.50 \mathrm{e} \AA^{-3}$

$\Delta \rho_{\min }=-0.43 \mathrm{e}^{-3}$ 
Refinement. All the $\mathrm{H}$ atoms were placed in their calculated positions and then refined using a riding model with bond lengths of $0.93 \AA(\mathrm{CH})$ or $0.97 \AA(\mathrm{CH} 2)$. Isotropic displacement parameters for all these atoms were set to $1.2(\mathrm{CH}, \mathrm{CH} 2)$ times $U_{\text {eq }}$ of the parent atom.

Fractional atomic coordinates and isotropic or equivalent isotropic displacement parameters $\left(\AA^{2}\right)$

\begin{tabular}{lllll}
\hline & $x$ & $y$ & $z$ & $U_{\text {iso }}{ }^{*} U_{\text {eq }}$ \\
\hline Br1 & $0.11401(3)$ & $0.16664(2)$ & $0.36163(5)$ & $0.05288(15)$ \\
O1 & $0.6397(2)$ & $0.49748(14)$ & $0.1531(4)$ & $0.0559(6)$ \\
O2 & $0.4267(3)$ & $0.56084(13)$ & $0.3097(4)$ & $0.0620(7)$ \\
N1 & $0.5551(2)$ & $0.37741(14)$ & $0.1875(3)$ & $0.0396(5)$ \\
N2 & $0.3293(2)$ & $0.44352(13)$ & $0.3284(3)$ & $0.0363(5)$ \\
N3 & $0.4718(2)$ & $0.25464(14)$ & $0.2338(3)$ & $0.0402(5)$ \\
C1 & $0.5508(3)$ & $0.45577(17)$ & $0.2002(4)$ & $0.0416(6)$ \\
C2 & $0.4292(3)$ & $0.49169(17)$ & $0.2831(4)$ & $0.0419(6)$ \\
C3 & $0.3366(2)$ & $0.36362(15)$ & $0.3063(4)$ & $0.0325(5)$ \\
C4 & $0.2339(3)$ & $0.31484(16)$ & $0.3433(4)$ & $0.0370(5)$ \\
H4 & 0.1537 & 0.3342 & 0.3800 & $0.044^{*}$ \\
C5 & $0.2537(3)$ & $0.23636(15)$ & $0.3243(4)$ & $0.0373(5)$ \\
C6 & $0.3740(3)$ & $0.20755(16)$ & $0.2754(4)$ & $0.0411(6)$ \\
H6 & 0.3869 & 0.1545 & 0.2715 & $0.049 *$ \\
C7 & $0.4528(3)$ & $0.32983(15)$ & $0.2449(4)$ & $0.0347(5)$ \\
C8 & $0.2161(3)$ & $0.47795(16)$ & $0.4219(4)$ & $0.0422(6)$ \\
H8A & 0.2010 & 0.4495 & 0.5379 & $0.051^{*}$ \\
H8B & 0.2379 & 0.5306 & 0.4578 & $0.051^{*}$ \\
C9 & $0.0952(3)$ & $0.47845(17)$ & $0.3036(5)$ & $0.0436(6)$ \\
C10 & $-0.0066(3)$ & $0.4782(2)$ & $0.2184(5)$ & $0.0535(8)$ \\
H10 & -0.0871 & 0.4780 & 0.1511 & $0.064^{*}$ \\
C11 & $0.6748(3)$ & $0.34154(19)$ & $0.1177(5)$ & $0.0507(7)$ \\
H11A & 0.6521 & 0.2944 & 0.0499 & $0.061^{*}$ \\
H11B & 0.7160 & 0.3762 & 0.0297 & $0.061^{*}$ \\
C12 & $0.7673(3)$ & $0.32418(18)$ & $0.2785(6)$ & $0.0524(8)$ \\
C13 & $0.8389(4)$ & $0.3114(3)$ & $0.4095(7)$ & $0.0715(11)$ \\
H13 & 0.8958 & 0.3012 & 0.5135 & $0.086^{*}$ \\
& & & &
\end{tabular}

Atomic displacement parameters $\left(\AA^{2}\right)$

\begin{tabular}{lllllll}
\hline & $U^{11}$ & $U^{22}$ & $U^{33}$ & $U^{12}$ & $U^{13}$ & $U^{23}$ \\
\hline Br1 & $0.0547(2)$ & $0.0393(2)$ & $0.0645(2)$ & $-0.01276(13)$ & $0.00117(15)$ & $0.00276(13)$ \\
O1 & $0.0480(12)$ & $0.0521(13)$ & $0.0681(15)$ & $-0.0138(10)$ & $0.0080(10)$ & $0.0030(11)$ \\
O2 & $0.0639(14)$ & $0.0322(11)$ & $0.0909(19)$ & $-0.0061(10)$ & $0.0152(13)$ & $-0.0050(11)$ \\
N1 & $0.0349(11)$ & $0.0403(12)$ & $0.0438(13)$ & $0.0003(9)$ & $0.0039(9)$ & $-0.0007(10)$ \\
N2 & $0.0386(11)$ & $0.0293(10)$ & $0.0410(12)$ & $0.0025(9)$ & $0.0029(9)$ & $-0.0028(9)$ \\
N3 & $0.0409(12)$ & $0.0361(11)$ & $0.0433(12)$ & $0.0066(9)$ & $-0.0015(9)$ & $-0.0036(10)$ \\
C1 & $0.0403(14)$ & $0.0433(15)$ & $0.0411(14)$ & $-0.0040(12)$ & $-0.0014(11)$ & $0.0012(12)$ \\
C2 & $0.0422(14)$ & $0.0343(14)$ & $0.0491(16)$ & $-0.0040(11)$ & $0.0006(11)$ & $-0.0014(11)$ \\
C3 & $0.0363(12)$ & $0.0305(12)$ & $0.0306(12)$ & $0.0012(10)$ & $-0.0014(9)$ & $0.0015(10)$ \\
C4 & $0.0371(13)$ & $0.0359(13)$ & $0.0380(13)$ & $0.0023(11)$ & $0.0020(10)$ & $-0.0004(11)$
\end{tabular}




\begin{tabular}{lllllll} 
C5 & $0.0449(14)$ & $0.0310(13)$ & $0.0357(13)$ & $-0.0036(11)$ & $-0.0029(10)$ & $0.0014(10)$ \\
C6 & $0.0495(15)$ & $0.0290(13)$ & $0.0443(15)$ & $0.0032(11)$ & $-0.0052(12)$ & $-0.0022(11)$ \\
C7 & $0.0356(12)$ & $0.0340(13)$ & $0.0341(13)$ & $0.0013(10)$ & $-0.0020(9)$ & $-0.0020(10)$ \\
C8 & $0.0473(15)$ & $0.0339(13)$ & $0.0460(15)$ & $0.0052(11)$ & $0.0071(12)$ & $-0.0077(11)$ \\
C9 & $0.0450(16)$ & $0.0376(14)$ & $0.0491(16)$ & $0.0085(11)$ & $0.0107(12)$ & $0.0009(12)$ \\
C10 & $0.0498(18)$ & $0.0539(18)$ & $0.0571(19)$ & $0.0096(14)$ & $0.0045(14)$ & $-0.0032(15)$ \\
C11 & $0.0410(15)$ & $0.0528(18)$ & $0.0590(19)$ & $0.0034(12)$ & $0.0115(13)$ & $-0.0033(14)$ \\
C12 & $0.0348(14)$ & $0.0452(16)$ & $0.078(2)$ & $-0.0002(12)$ & $0.0109(15)$ & $0.0023(15)$ \\
C13 & $0.0465(19)$ & $0.074(3)$ & $0.094(3)$ & $0.0025(18)$ & $-0.004(2)$ & $0.012(2)$ \\
\hline
\end{tabular}

Geometric parameters $\left(A,{ }^{\circ}\right)$

\begin{tabular}{|c|c|c|c|}
\hline $\mathrm{Br} 1-\mathrm{C} 5$ & $1.894(3)$ & $\mathrm{C} 4-\mathrm{H} 4$ & 0.9300 \\
\hline $\mathrm{O} 1-\mathrm{C} 1$ & $1.217(4)$ & $\mathrm{C} 4-\mathrm{C} 5$ & $1.384(4)$ \\
\hline $\mathrm{O} 2-\mathrm{C} 2$ & $1.214(4)$ & $\mathrm{C} 5-\mathrm{C} 6$ & $1.381(4)$ \\
\hline $\mathrm{N} 1-\mathrm{C} 1$ & $1.363(4)$ & C6- $-\mathrm{H} 6$ & 0.9300 \\
\hline $\mathrm{N} 1-\mathrm{C} 7$ & $1.402(4)$ & $\mathrm{C} 8-\mathrm{H} 8 \mathrm{~A}$ & 0.9700 \\
\hline $\mathrm{N} 1-\mathrm{C} 11$ & $1.472(4)$ & $\mathrm{C} 8-\mathrm{H} 8 \mathrm{~B}$ & 0.9700 \\
\hline $\mathrm{N} 2-\mathrm{C} 2$ & $1.365(4)$ & $\mathrm{C} 8-\mathrm{C} 9$ & $1.457(5)$ \\
\hline $\mathrm{N} 2-\mathrm{C} 3$ & $1.397(3)$ & $\mathrm{C} 9-\mathrm{C} 10$ & $1.176(5)$ \\
\hline $\mathrm{N} 2-\mathrm{C} 8$ & $1.477(3)$ & $\mathrm{C} 10-\mathrm{H} 10$ & 0.9300 \\
\hline N3-C6 & $1.331(4)$ & $\mathrm{C} 11-\mathrm{H} 11 \mathrm{~A}$ & 0.9700 \\
\hline $\mathrm{N} 3-\mathrm{C} 7$ & $1.322(4)$ & $\mathrm{C} 11-\mathrm{H} 11 \mathrm{~B}$ & 0.9700 \\
\hline $\mathrm{C} 1-\mathrm{C} 2$ & $1.524(4)$ & $\mathrm{C} 11-\mathrm{C} 12$ & $1.472(5)$ \\
\hline $\mathrm{C} 3-\mathrm{C} 4$ & $1.379(4)$ & $\mathrm{C} 12-\mathrm{C} 13$ & $1.172(6)$ \\
\hline $\mathrm{C} 3-\mathrm{C} 7$ & $1.405(4)$ & $\mathrm{C} 13-\mathrm{H} 13$ & 0.9300 \\
\hline $\mathrm{C} 1-\mathrm{N} 1-\mathrm{C} 7$ & $122.8(2)$ & $\mathrm{N} 3-\mathrm{C} 6-\mathrm{C} 5$ & $120.9(2)$ \\
\hline $\mathrm{C} 1-\mathrm{N} 1-\mathrm{C} 11$ & $118.2(2)$ & N3-C6-H6 & 119.5 \\
\hline $\mathrm{C} 7-\mathrm{N} 1-\mathrm{C} 11$ & $118.9(2)$ & $\mathrm{C} 5-\mathrm{C} 6-\mathrm{H} 6$ & 119.5 \\
\hline $\mathrm{C} 2-\mathrm{N} 2-\mathrm{C} 3$ & $122.5(2)$ & $\mathrm{N} 1-\mathrm{C} 7-\mathrm{C} 3$ & $119.3(2)$ \\
\hline $\mathrm{C} 2-\mathrm{N} 2-\mathrm{C} 8$ & $117.4(2)$ & $\mathrm{N} 3-\mathrm{C} 7-\mathrm{N} 1$ & $116.8(2)$ \\
\hline $\mathrm{C} 3-\mathrm{N} 2-\mathrm{C} 8$ & $119.8(2)$ & $\mathrm{N} 3-\mathrm{C} 7-\mathrm{C} 3$ & $123.9(3)$ \\
\hline $\mathrm{C} 7-\mathrm{N} 3-\mathrm{C} 6$ & $118.7(2)$ & $\mathrm{N} 2-\mathrm{C} 8-\mathrm{H} 8 \mathrm{~A}$ & 108.8 \\
\hline $\mathrm{O} 1-\mathrm{C} 1-\mathrm{N} 1$ & $123.3(3)$ & $\mathrm{N} 2-\mathrm{C} 8-\mathrm{H} 8 \mathrm{~B}$ & 108.8 \\
\hline $\mathrm{O} 1-\mathrm{C} 1-\mathrm{C} 2$ & $119.2(3)$ & $\mathrm{H} 8 \mathrm{~A}-\mathrm{C} 8-\mathrm{H} 8 \mathrm{~B}$ & 107.7 \\
\hline $\mathrm{N} 1-\mathrm{C} 1-\mathrm{C} 2$ & $117.5(2)$ & $\mathrm{C} 9-\mathrm{C} 8-\mathrm{N} 2$ & $114.0(2)$ \\
\hline $\mathrm{O} 2-\mathrm{C} 2-\mathrm{N} 2$ & $123.2(3)$ & $\mathrm{C} 9-\mathrm{C} 8-\mathrm{H} 8 \mathrm{~A}$ & 108.8 \\
\hline $\mathrm{O} 2-\mathrm{C} 2-\mathrm{C} 1$ & $119.0(3)$ & $\mathrm{C} 9-\mathrm{C} 8-\mathrm{H} 8 \mathrm{~B}$ & 108.8 \\
\hline $\mathrm{N} 2-\mathrm{C} 2-\mathrm{C} 1$ & $117.7(2)$ & $\mathrm{C} 10-\mathrm{C} 9-\mathrm{C} 8$ & $175.8(3)$ \\
\hline $\mathrm{N} 2-\mathrm{C} 3-\mathrm{C} 7$ & $119.9(2)$ & $\mathrm{C} 9-\mathrm{C} 10-\mathrm{H} 10$ & 180.0 \\
\hline $\mathrm{C} 4-\mathrm{C} 3-\mathrm{N} 2$ & $122.9(2)$ & $\mathrm{N} 1-\mathrm{C} 11-\mathrm{H} 11 \mathrm{~A}$ & 109.6 \\
\hline $\mathrm{C} 4-\mathrm{C} 3-\mathrm{C} 7$ & $117.3(2)$ & $\mathrm{N} 1-\mathrm{C} 11-\mathrm{H} 11 \mathrm{~B}$ & 109.6 \\
\hline $\mathrm{C} 3-\mathrm{C} 4-\mathrm{H} 4$ & 121.0 & $\mathrm{H} 11 \mathrm{~A}-\mathrm{C} 11-\mathrm{H} 11 \mathrm{~B}$ & 108.1 \\
\hline $\mathrm{C} 3-\mathrm{C} 4-\mathrm{C} 5$ & $118.1(2)$ & $\mathrm{C} 12-\mathrm{C} 11-\mathrm{N} 1$ & $110.1(3)$ \\
\hline $\mathrm{C} 5-\mathrm{C} 4-\mathrm{H} 4$ & 121.0 & $\mathrm{C} 12-\mathrm{C} 11-\mathrm{H} 11 \mathrm{~A}$ & 109.6 \\
\hline $\mathrm{C} 4-\mathrm{C} 5-\mathrm{Br} 1$ & $120.1(2)$ & $\mathrm{C} 12-\mathrm{C} 11-\mathrm{H} 11 \mathrm{~B}$ & 109.6 \\
\hline $\mathrm{C} 6-\mathrm{C} 5-\mathrm{Br} 1$ & $118.9(2)$ & $\mathrm{C} 13-\mathrm{C} 12-\mathrm{C} 11$ & $178.3(4)$ \\
\hline
\end{tabular}


data reports

$\begin{array}{llll}\mathrm{C} 6-\mathrm{C} 5-\mathrm{C} 4 & 121.0(3) & \mathrm{C} 12-\mathrm{C} 13-\mathrm{H} 13 & 180.0\end{array}$

Hydrogen-bond geometry $\left(\AA,{ }^{\circ}\right)$

\begin{tabular}{lllll}
\hline$D-\mathrm{H} \cdots A$ & $D-\mathrm{H}$ & $\mathrm{H} \cdots A$ & $D \cdots A$ & $D-\mathrm{H}^{\cdots} \cdot A$ \\
\hline $\mathrm{C} 6-\mathrm{H} 6 \cdots \mathrm{O} 2^{\mathrm{i}}$ & 0.93 & 2.58 & $3.326(4)$ & 137 \\
\hline
\end{tabular}

Symmetry code: (i) $-x+1, y-1 / 2,-z+1 / 2$. 